\title{
Comparison of SPEA2 and NSGA-II Applied to Automatic Inventory Control System Using Hypervolume Indicator
}

\author{
Ewelina CHOŁODOWICZ, Przemysław ORŁOWSKI \\ West Pomeranian University of Technology Szczecin, \\ Sikorskiego 37, Szczecin, 70-313, Poland. \\ cholodowicz.ewelina@gmail.com \\ przemyslaw.orlowski@zut.edu.pl
}

\begin{abstract}
The optimization of multi-objective problems is an area of important research. The importance attained by this type of problems has allowed the development of multiple algorithms. To determine which multi-objective algorithm has the best performance with respect to the problem of goods flow in the inventory, in this article an experimental comparison between two of the main multi-objective evolutionary algorithms is conducted: Nondominated Sorting Genetic Algorithm II (NSGA-II) and Strength Pareto Evolutionary Algorithm 2 (SPEA2). The inventory model is optimized by taking into account two objectives: minimal cost of lost opportunities to make sales and minimal cost of used space in the inventory. The results obtained by both algorithms are compared and analysed based on hypervolume indicator that measures the volume of the dominated space.
\end{abstract}

Keywords: inventory control system; SPEA2; NSGA-II; multi-objective optimization; hypervolume.

\section{Introduction}

Growing inventory management complexity is making it increasingly difficult to effectively managed inventories. The objective of inventory management is to provide uninterrupted production, sales, customerservice levels at the minimum cost. Inventory control is about generating maximum profits with minimum inventory investment, without affecting customer satisfaction levels. Due to effective inventory control, well-received products can always be delivered, going out of stock can be avoided. There has been an growing interest in solving inventory management problem. More and more works have been focusing upon inventory control systems: [23],[21],[22],[3]. Moreover, lead time uncertainty can disrupt the proper performance of such systems. Therefore, papers are also continuously dealing with uncertainty in inventory control [8], [24]. As a result of the emergence of complex inventory control systems, scientist began to use the methods of multi-criteria optimization: [25],[14],[16],[19].

Multi-objective optimization problems appear in many fields, such as engineering, economics, and logistics. In the last decade, evolutionary approaches have been the primary tools to solve real-world multi-objective problems. Multi-objective optimization algorithms allow optimization performance by taking into account multiple objectives simultaneously and searching for the set of optimal solutions that form the socalled Pareto front. Evolutionary multi-objective optimization, which applies evolutionary computation to multi-objective optimization, has attracted a great deal of attention recently [6],[20],[9],[12] There are many varieties of multi-objective genetic algorithms (MOGA), but the best studied is the application of genetic algorithms (GA) to multi-objective optimization problem. In multiobjective optimization, the problem is to find best possible compromise solutions which cannot be improved according to one objective without deteriorating the other. Nowadays, there is a great range of algorithms and their applications in a variety of multi-objective problems. Among the multi-objective, genetic algorithms, SPEA2 [29] proposed by Zitzler and NSGA-II [7] proposed by Deb provide excellent results as compared with other multiobjective genetic algorithms proposed. These algorithms include important search mechanisms, such as preservation of good solutions discovered in the search and appropriate reduction of the possible Paretooptimal solutions [13].

The design of quality measures for approximations of the Pareto-optimal set is of high importance not only for the performance assessment, but also for the construction of 
multi-objective optimizers [30]. To evaluate and compare the set of solutions found by these optimizers, quality indicators have been developed. A popular way to measure the quality of a Pareto set is the hypervolume indicator which measures the volume of the dominated space bounded from below by a reference point [1]. The hypervolume indicator was first proposed in [31],[32] and was called as 'size of the space covered', and in other works as 'hyperarea metric'[26], 'S-metric' [33] and 'Lebesgue measure' [17],[11]. Using the hypervolume of the dominated portion of the objective space as a measure for the quality of Pareto set approximations has received more and more attention in recent years. The hypervolume indicator is one of the most popular measures for the performance assessment of multi-objective optimizers and in this context it has been subject to several investigations [28],[18],[10]. There are some studies that discuss the usage of this measure for multi-objective search [34],[15],[2] and in particular the issue of fast hypervolume calculation. Moreover, this indicator has been successfully integrated into stochastic optimizers, such as evolutionary algorithms, where it serves as a guidance criterion for searching the parameter space.

Main aim of the paper is to compare performances of two evolutionary multiobjective algorithms (SPEA2 and NSGA-II) applied to automatic inventory control system. To perform the comparison, the hypervolume indicator is calculated for sets of Pareto fronts.

\section{Model for solving optimization problem}

Model of an inventory control system from [4] is used as a tool to calculate the performance of optimization algorithms. The block diagram of the analysed system is shown in Fig.1.

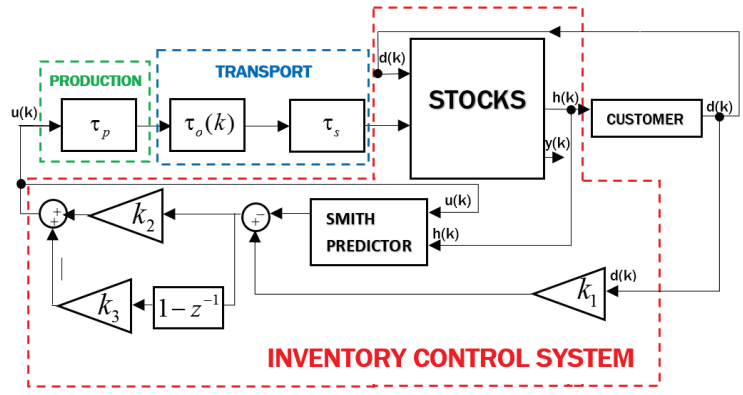

Figure 1. Block diagram of inventory system with control.
As far as inventory systems are concerned, inventory can be represented as plant with two inputs: $u(k)$ (supply) which increases stocks and $d(k)$ (time-varying demand for products from the inventory) which is limited $0 \leq h(k) \leq d(k) \leq d_{\max }$, and two outputs: $h(k)$ (finished products delivered to customers) and $y(k)$ (the level of stock). This model does not include returns and has a limit of maximum demand and the second output. From the logistics point of view, the inventory is a buffer of goods flow, so it accumulates delivered products. The system consists of three main parts: production, transport and storage. Time-dependent delay $\tau_{o}(k)$ is associated with waiting for a mean of transport and accumulation of orders. Assuming that the delays $\tau_{p}, \tau_{s}$ are known and function $q(k)$ for $k \in \mathrm{N}$ this model is a linear, non-stationary, discrete dynamical system model. The product quantity stored in the inventory at moment $k$, called the stock, is therefore given as follows:

$$
y(k)=y(k-1)+(1-q(k)) x\left(k-\tau_{s}\right)-h(k)(1)
$$

The product quantity awaiting shipment at moment $k$ is defined by the ollowing relationship:

$x(k)=q(k-1) x(k-1)+u\left(k-\tau_{p}\right)$

Time-varying delay is implemented in form of $q(k)$, which is shipping rate at moment $k$ defined as follows:

$q(k)=\left\{\begin{array}{l}0-\text { shipment } \\ 1 \text { - waiting for transport }\end{array}\right.$

Inventory control system consists of Smith predictor (It is based on implementation of the model without delay and with delay) and discrete-time proportional- derivative controller (PD controller) and is described in [4]. Model of the system without delay, implemented in the Smith predictor is given in the form:

$\hat{y}(k)=\hat{y}(k-1)+u(k-1)-h(k)$

Discrete-time proportional-derivative controller is given in the following form: 


$$
u(k)=k_{2} \varepsilon(k)+k_{3}(\varepsilon(k)-\varepsilon(k-1))
$$

where:

$$
\begin{aligned}
& \varepsilon(k)=y_{\text {ref }}(k)-\hat{y}(k) \\
& y_{\text {ref }}(k)=k_{1} d(k)
\end{aligned}
$$

In the system presented in fig. 1, optimization task determines the problem of finding the optimal values of the parameters $k_{i}(i=1,2,3)$. Cost functions are presented by following relations:

$$
\begin{aligned}
& j_{1}=\frac{1}{N} \sum_{k=n_{0}}^{N}(d(k)-h(k)) \\
& j_{2}=\frac{1}{N} \sum_{k=n_{0}}^{N} y(k)
\end{aligned}
$$

Time required to deliver ordered products to the inventory for $k=0$ is $n_{0}=\tau_{p}+\tau_{o}\left(\tau_{p}\right)+\tau_{s}$ and $N$ is the length of the time horizon. The equation (8) represents cost of shortages. In turn, the expression (9) represents holding cost. These indicators are associated with financial costs. Its form is based on the physical interpretation of the problem and can be evaluated in arbitrary currency. The objective is presented as the following vector:

$$
\mathbf{j}=\left[j_{1}, j_{2}\right]
$$

For the model and control system described in $[28,29]$ and the quality indicator in the form of (8) - (10) the optimization problem can be defined in the following form:

$$
\min _{\mathbf{k}} \mathbf{j}
$$

\section{Algorithms NSGA-II and SPEA2}

In some areas such as engineering or economics, often have problems that require simultaneous optimization of two or more functions, in these cases, there is talk of multi-objective optimization problems. Evolutionary Algorithms have become popular as robust and effective methods for solving optimization problems. The Nondominated Sorting Genetic Algorithm and SPEA2 is a multi-objective algorithm and is an instance of an Evolutionary Algorithm from the field of Evolutionary Computation.

\section{A. NSGA-II}

There are two versions of the algorithm, the classical NSGA and the updated and currently canonical form NSGA-II. The Non-dominated Sorting Genetic Algorithm II (NSGA-II) is proving to be a robust optimization algorithm for a whole range of multi-objective problems. The non-dominated sorting segregates the population

By first finding the non-dominated solutions in

the population, and marks these points as the first

front. In the next step calculates the average distance between members of each front on the front itself. Parents are selected from the population by using binary tournament selection based on the rank and crowding distance. An individual is selected if the rank is smaller than the other or if crowding distance is greater than the other. Crowding distance is compared only if ranks for both individuals are the same. The selected population generates offsprings from crossover and mutation operators. The population with the current population and current offsprings is sorted again based on non-domination and only the best individuals are selected taking into account population size. The selection is based on the rank and on the crowding distance on the last front. In a nutshell, NSGA-II algorithm has three features: it uses an elitist principle, emphasizes non-dominated solutions, uses an explicit diversity preserving mechanism [6].

\section{B. SPEA2}

The improved Strength Pareto Evolutionary Approach (SPEA2) is chosen to perform the control system optimization resulting in the final analysis and comparison. SPEA is an extension of the Genetic Algorithm for multiple objective optimization problems. SPEA2 has an external archive consisting of the previously found nondominated solutions. It is updated after each generation and for each solution a strength value is computed. An archive of the non-dominated set is maintained separate from the population of candidate solutions used in the evolutionary process, providing a form of elitism. Due to potential weaknesses of SPEA, the improved version - SPEA2 has better fitness assignment scheme, more precise guidance of the search and a new archive truncation methods. To avoid situations where population members dominated by the same members of the archive have the 
same fitness value, SPEA2 takes into account both the number of dominating and dominated solutions in computing the raw fitness of a solution. The objective of the algorithm is to locate and maintain a front of non-dominated solutions - set of Pareto optimal solutions. This is achieved by using evolutionary process to explore the search space, and a selection process that uses a combination of the degree to which a candidate solution is dominated and an estimation of density of the Pareto front as an assigned fitness.

An archive of the non-dominated set is kept separate from the population of candidate solutions used in the evolutionary process, which represents a kind of elitism. [29].

\section{Simulation and results}

In this section the results of computer simulations and an experimental comparison between two algorithms: SPEA2 and NSGA-II for multi-objective optimization is presented. To evaluate the performance of the algorithms, we use hypervolume indicator with reference point whose value was estimated on the basis of the spread of points on all Pareto fronts and equal: $r=\left[\begin{array}{ll}350 & 25000\end{array}\right]$. The reference point is placed in such a way as to be dominated by most members of the sets to be investigated. Initial conditions of SPEA2 and NSGA-II are presented in the table 1.

Table 1. Parameters of the algorithms

\begin{tabular}{|c|c|}
\hline Parameter & SPEA2 and NSGA-II \\
\hline Number of generations & {$[1,400]$} \\
\hline Population size & $5,10,20,40,80$ \\
\hline Mutation probability & 0.4 \\
\hline Crossover probability & 0.7 \\
\hline Mutation rate & 0.02 \\
\hline
\end{tabular}

The structure of the control system in fig. 1 is applied as an optimization model with production delay and shipping delay: $\tau_{p}=\tau_{s}=8$, time horizon: $N=1000$ days and the sampling period: 1 day. Time-varying market demand function $d(k)$ shown in fig. 2 is taken as an input signal to the inventory system.

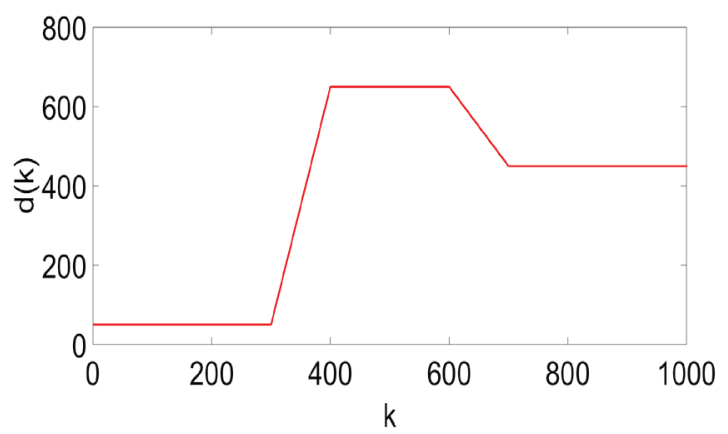

Figure 2. Presumed function of market demand $d(k)$ for products.

Results for SPEA2 are marked by red line and for NSGA-II by blue line. On the basis of the results we try to evaluate: which algorithm has better performance for the proposed model of inventory control system.

First, HYP is calculated for each of the 100 iterations of algorithms. Results are presented in fig 2 and 3 . The hypervolume metric assesses the area that is between a chosen reference point (corner to a hypercube), and the Pareto surface which limits the hypercube. The hypervolume indicator assesses not only the proximity to the true Pareto front, but also the diversity of solutions obtained across the set, especially at the edges. The higher value of hypervolume indicator, the better Pareto set.

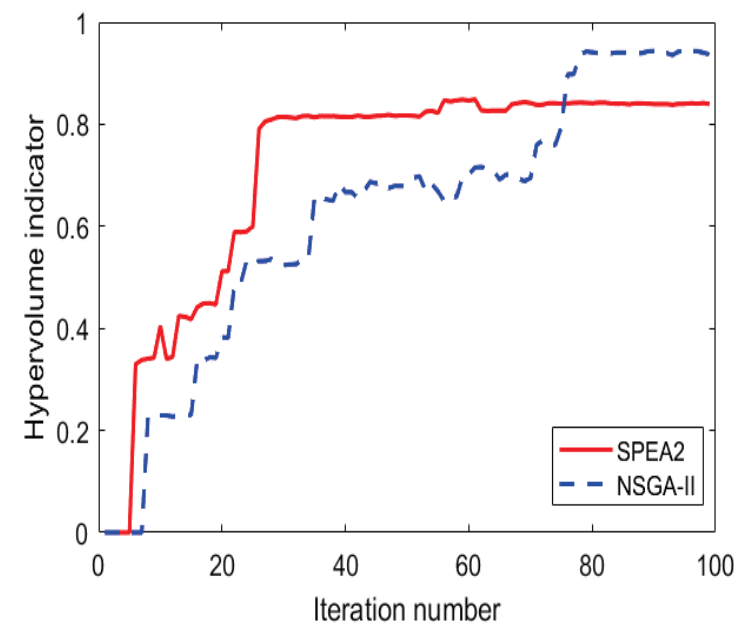

Figure 3. Hypervolume indicator vs. iterations for population size: 10 .

It can be seen from fig. 2 and 3 that hypervolume value (HYP) is increasing with the increase of the iteration for both algorithms. However, according to fig. 2 SPEA2 has the 
advantage over NSGA-II to about 75 iteration because achieves higher values of HYP.

Interesting observation is that HYP is always lower for NSGA-II at first, but later becomes higher. SPEA2 hold almost the same HYP from iteration number 25 , but NSGA-II increases it slowly and hold the highest value from iteration number 75 to 100 so at the last iteration has the best result. SPEA2 has the better ability to fast finding good Pareto set, however it quickly loses diversity of founded solutions for small number of iterations and in the last iteration has worse solutions than NSGA-II.

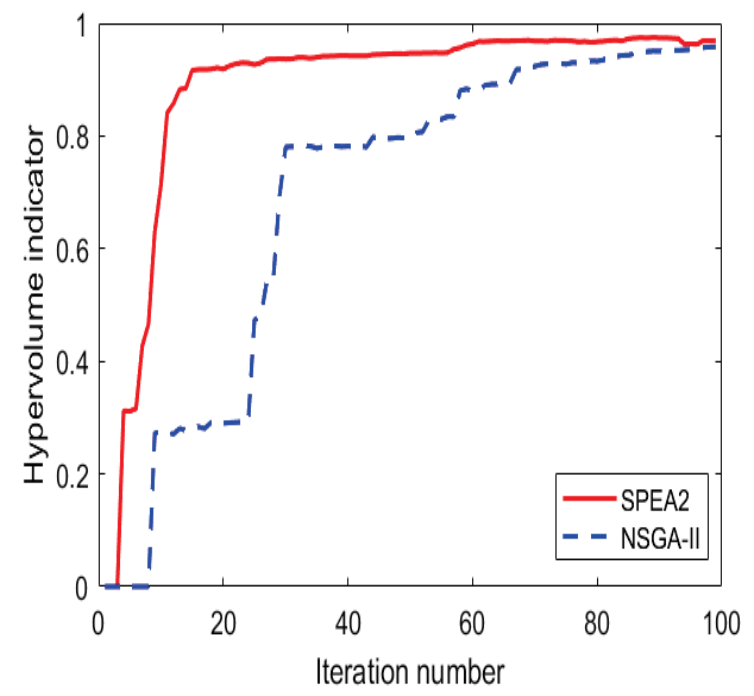

Figure 4. Hypervolume indicator vs. iterations for population size: 80 .

One the other hand, for situation in which the number of individuals in population is 8 -fold higher, as in fig. 4, SPEA2 achieves higher value of the HYP for all iterations and also has the ability to quickly find the Pareto front with the high value of HYP. For example, starting from about 20 iteration SPEA2 reaches almost the same value (0.96) as in the last iteration, and what is more, it is higher about 4 times than for NSGA-II (0.22). The results show clearly that SPEA2 outperforms NSGA-II in every iteration when the population size is higher. The hypervolume shows the speed of the convergence of the algorithms so SPEA2 converges faster to a better Pareto front approximation and obtains very good results quickly while NSGA-II require more time to reach the same quality of Pareto set.

Influence of the population size can be seen also in fig 4 and 5. Several simulations were performed in order to investigate the influence of the population size as well as the maximum number of generations converging towards the Pareto-optimal front. In fig. 4 and 5, the outcomes of each iterations for NSGA-II and SPEA2 are visualized in 3D space. For small number of population better performance has SPEA2 and is able to find Pareto-optimal set regarding the small population size. NSGA-II for small population has HYP close to zero, when SPEA2 is able to reach significantly higher HYP values.

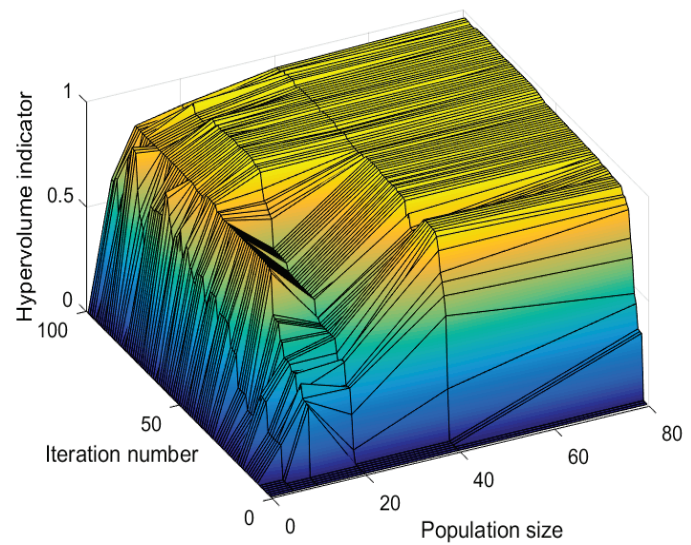

Figure 5. Hypervolume indicator for SPEA2: 1 to 100 iterations for population sizes: $5,10,20,40,80$.

As can be seen on fig 5, SPEA2 keeps the local minimum, and needs about 70 iterations to reach the highest values of HYP. What is more, SPEA2 has even the highest HYP for small number of iterations (smaller than 10) when population size is higher than 40 .

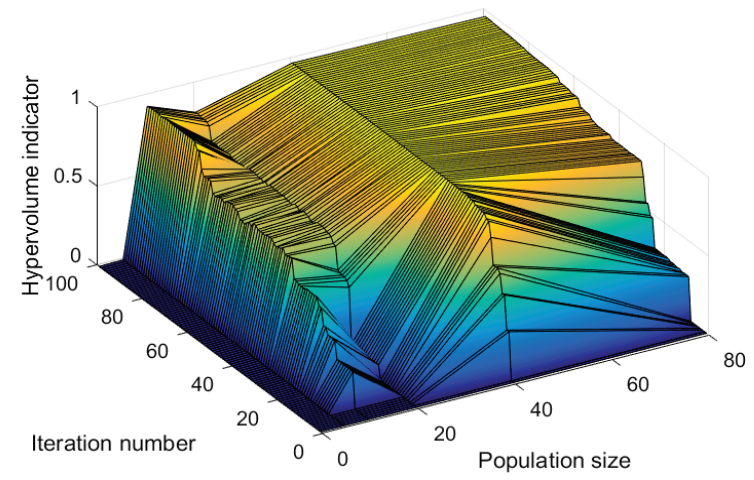

Figure 6. Hypervolume indicator for NSGA-II: 1 to 100 iterations for population sizes: 5, 10, 20, 40, 80 .

On the other hand, fig. 6 shows that NSGA-II is not able to achieve as good performance as SPEA2 for small number of iterations even for large population size (about 80 ). NSGA-II has the best results for both large population (more 
than 40 individuals) and high number of iterations (over 40). Furthermore, what is interesting that HYP of NSGA-II equals almost 0 HYP for population of 5 individuals, despite of increasing number of iterations when SPEA2 at the same scenario has significantly higher HYP.

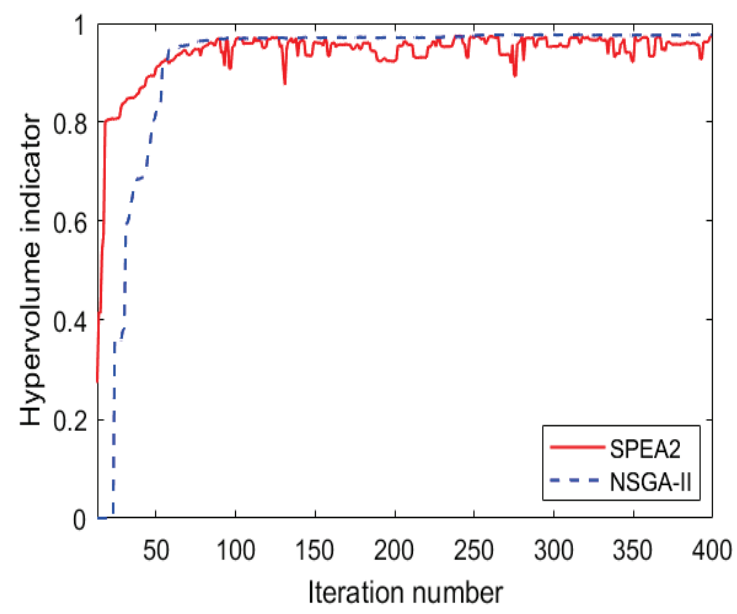

Figure 7. Hypervolume indicator: 1 to 400 iterations for population size: 40 .

Fig 7 indicates that both algorithms show comparable results in target of the presented optimization task. The difference in search ability is significantly visible between SPEA2 and NSGA-II maybe because of the fact that congestion is considered in binary tournament selection in NSGA-II mating selection. In tournament selection in NSGA-II, when the fitness values of two individuals being compared are equal, the individual with lower congestion is selected. Therefore, it can produce a more uniformly distributed population than SPEA2 [27].

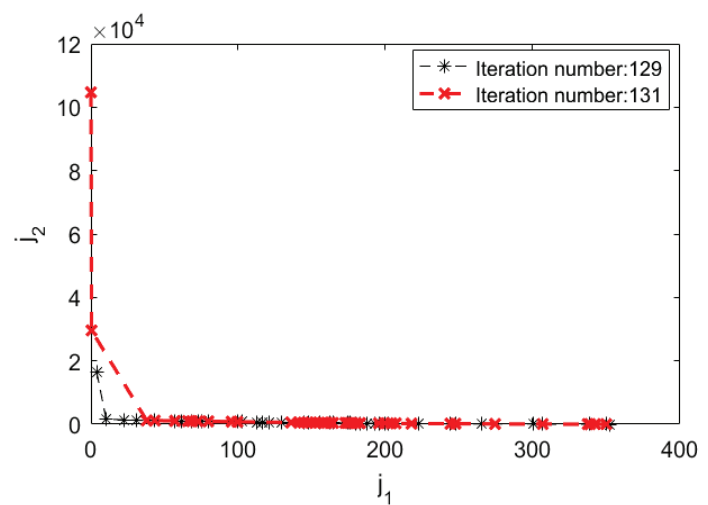

Figure 8. Pareto Fronts for SPEA2 which show case with a sharp decline in the value of the hypervolume indicator from fig. 7 .

Although the differences in performance appear large when population size is small, as fig. 5 and 6 show, the difference is no so distinct when it comes to larger population and higher number of iterations, as fig. 7 shows. In the next step, impact studies were conducted with a higher number of iterations. In this way, significant difference between algorithms can be seen in fig. 7. It can be seen that SPEA2 has a tendency to decrease HYP and it fluctuates up and down visibly for certain iterations. To look more closely at the reasons for the behavior of SPEA2, Pareto fronts in fig. 8 are presented.

As can be seen, SPEA2 removes solution close to the point of origin to expand range of solution set in iteration 131. This behavior is caused by a nearest neighbor density estimation technique which increases diversity.

\section{Conclusions}

In this paper, we have performed an experimental comparison between two algorithms for multi-objective optimization: SPEA2 and NSGA-II. Comparison was based on hypervolume indicator borrowed from the literature. It has been found that for the automatic inventory control system, SPEA2 outperforms NSGA-II for considered scenarios. SPEA2 is better than the NSGA-II when population size and number of iterations is small. Obviously, small populations do not provide enough diversity among the individuals. Increasing the population size, however, does not automatically cause an increase in performance, as can be observed in performance of NSGA-II for small iteration number. SPEA2 is able to faster finding the best Pareto front, but quality of this Pareto front mostly depends on number of iterations. Although, when number of iterations and individuals is high, performances of both algorithms are similar. These observation suggest that SPEA2 and NSGA-II are effective algorithms for presented kind of problem.

\section{REFERENCES}

1. Bringmann, K., \& Friedrich, T. (2013). Approximation quality of the hypervolume indicator. Artificial Intelligence, 195, pp. 265-290.

2. Chołodowicz, E., \& Orłowski, P. (2015). Dynamiczny dyskretny model systemu magazynowego ze zmiennym w czasie opóźnieniem. Logistyka, 4, pp. 28-32.

3. Chołodowicz, E., \& Orłowski, P. (2015). A periodic inventory control system with 
adaptive reference stock level for long supply delay. Measurement Automation Monitoring, 61, pp. 568-572.

4. Chołodowicz, E., \& Orłowski, P. (2015). Sterowanie przepływem towarów w magazynie $\mathrm{z}$ wykorzystaniem predyktora Smitha. Pomiary Automatyka Robotyka, 19(3), pp. 55-60.

5. Chołodowicz, E. \& Orłowski, P. (2016). Comparison of a Perpetual and PD Inventory Control System with Smith Predictor and Different Shipping Delays Using Bicriterial Optimization and SPEA2. Pomiary Automatyka Robotyka, 20(3), pp.5-12.

6. Deb, K. (2014). Multi-objective optimization. In Search methodologies Search methodologies. Introductory tutorials in optimization and decision support techniques Springer, pp. 403-449.

7. Deb, K., Agrawal, S., Pratap, A., \& Meyarivan, T. (2000). A fast elitist nondominated sorting genetic algorithm for multi-objective optimization: NSGA-II. In International Conference on Parallel Problem Solving From Nature, pp.849-858.

8. Dolgui, A., Ammar, O. B., Hnaien, F., \& Louly, M. A. (2013). A state of the art on supply planning and inventory control under lead time uncertainty. Studies in Informatics and Control, 22(3), pp. 255268.

9. Donoso, Y., \& Fabregat, R. (2007). Multiobjective optimization in computer networks using metaheuristics. Auerbach Publications, Taylor \& Francis Group.

10. Emmerich, M., Beume, N., \& Naujoks, B. (2005). An EMO algorithm using the hypervolume measure as selection criterion. In International Conference on Evolutionary Multi-Criterion Optimization pp. 62-76.

11. Fleischer, M. (2003). The measure of Pareto optima applications to multiobjective metaheuristics. In International Conference on Evolutionary MultiCriterion Optimization, pp. 519-533.

12. Ghiasi, M. S., Arjmand, N., Boroushaki, M., \& Farahmand, F. (2016). Investigation of trunk muscle activities during lifting using a multi-objective optimization-based model and intelligent optimization algorithms. Medical \& biological engineering \& computing, 54(2-3), pp. 431-440.

13. Hiroyasu, T., Nakayama, S., \& Miki, M. (2005). Comparison study of SPEA2+, SPEA2, and NSGA-II in diesel engine emissions and fuel economy problem. In The 2005 IEEE Congress on Evolutionary Computation, pp. 236-242.

14. Hnaien, F., Delorme, X., \& Dolgui, A. (2010). Multi-objective optimization for inventory control in two-level assembly systems under uncertainty of lead times. Computers \& operations research, 37(11), pp. 1835-1843.

15. Knowles, J. D., Corne, D. W., \& Fleischer, M. (2003). Bounded archiving using the Lebesgue measure. In The 2003 Congress on Evolutionary Computation, pp. 2490-2497.

16. Lagos, C., Vega, J., Guerrero, G., \& Rubio, J. M. (2016). Solving a Novel MultiObjective Inventory Location Problem by means of a Local Search Algorithm. Studies in Informatics and Control , 25(2), pp. 189-194.

17. Laumanns, M., Rudolph, G., \& Schwefel, H. P. (1999). Approximating the pareto set: Concepts, diversity issues, and performance assessment. Technical Report, CI, pp. 72/99.

18. Le, K. N., \& Landa-Silva, D. (2016). Hyper-volume evolutionary algorithm. $V N U$ journal of science: computer science and communication engineering, 32(1), pp. 10-32.

19. Liao, S. H., Hsieh, C. L., \& Lai, P. J. (2011). An evolutionary approach for multi-objective optimization of the integrated location-inventory distribution network problem in vendor-managed inventory. Expert Systems with Applications, 38(6), pp. 6768-6776.

20. Luo, Z., Sultan, U., Ni, M., Peng, H., Shi, B., \& Xiao, G. (2016). Multi-objective optimization for GPU3 Stirling engine by combining multi-objective algorithms. Renewable Energy, 94, pp. 114-125. 
21. Maihami, R., \& Kamalabadi, I. N. (2012). Joint pricing and inventory control for noninstantaneous deteriorating items with partial backlogging and time and price dependent demand. International Journal of Production Economics, 136(1), pp. 116-122.

22. Mohammaditabar, D., Ghodsypour, S. H., \& O'Brien, C. (2012). Inventory control system design by integrating inventory classification and policy selection. International Journal of Production Economics, 140(2), pp. 655-659.

23. Mousavi, S. M., Hajipour, V., Niaki, S. T. A., \& Alikar, N. (2013). Optimizing multiitem multi-period inventory control system with discounted cash flow and inflation: two calibrated meta-heuristic algorithms. Applied Mathematical Modelling, 37(4), pp. 2241-2256.

24. Schmitt, A. J., \& Snyder, L. V. (2012). Infinite-horizon models for inventory control under yield uncertainty and disruptions. Computers \& Operations Research, 39(4), pp. 850-862.

25. Srivastav, A., \& Agrawal, S. (2016). Multiobjective optimization of hybrid backorder inventory model. Expert Systems with Applications, 51, pp. 76-84.

26. Van Veldhuizen, D. A. (1999). Multiobjective evolutionary algorithms: classifications, analyses, and new innovations. Ph. D. thesis, Graduate School of Engineering of the Air Force Institute of Technology.

27. Yao, X., Burke, E., Lozano, J. A., Smith, J., Merelo-Guervós, J. J., Bullinaria, J. A., \& Schwefel, H. P. (Eds.). (2004). In Parallel Problem Solving from NaturePPSN VIII. In 8th International Conference, pp. 18-22.
28. Yang, K., Deutz, A., Yang, Z., Back, T., \& Emmerich, M. (2016). Truncated expected hypervolume improvement: Exact computation and application. In IEEE Congress on Evolutionary Computation (CEC), pp. 4350-4357.

29. Zitzler, E., Laumanns, M., \& Thiele, L. (2001). SPEA2: Improving the strength Pareto evolutionary algorithm. In Proc. EUROGEN 2001. Evolutionary Methods for Design, Optimization and Control With Applications to Industrial Problems, pp. 95-100.

30. Zitzler, E., Brockhoff, D., \& Thiele, L. (2007). The hypervolume indicator revisited: On the design of Paretocompliant indicators via weighted integration. In International Conference on Evolutionary Multi-Criterion Optimization pp. 862-876.

31. Zitzler, E., \& Thiele, L. (1998). Multiobjective optimization using evolutionary algorithms - a comparative case study. In International Conference on Parallel Problem Solving from Nature, pp. 292-301.

32. Zitzler, E., \& Thiele, L. (1999). Multiobjective evolutionary algorithms: a comparative case study and the strength Pareto approach. IEEE transactions on Evolutionary Computation, 3(4), pp. 257-271.

33. Zitzler, E. (1999). Evolutionary algorithms for multiobjective optimization: Methods and applications. $\mathrm{PhD}$ thesis, Swiss Federal Institute of Technology (ETH) Zurich

34. Zitzler, E., \& Künzli, S. (2004). Indicatorbased selection in multiobjective search. In International Conference on Parallel Problem Solving from Nature, pp. 832-842. 